\title{
REDUCTION IN INITIAL BOUNDARY VALUE PROBLEM FOR HIV EVOLUTION MODEL
}

\author{
A. Archibasov \\ Samara National Research University, Samara, Russia
}

\begin{abstract}
HIV evolution model that describes the dynamics of concentrations of uninfected and infected cells is considered in the paper. The introduction of dimensionless variables and parameters leads to the initial boundary value problem for singularly perturbed system of partial integro-differential equations. By Tikhonov-Vasil'eva boundary functions method asymptotic solution with boundary layer is constructed. Numeric simulation of complete and reduced systems are also given.
\end{abstract}

Keywords: singular perturbations, asymptotic expansions, boundary layer, boundary functions.

Citation: Archibasov A. Reduction in initial boundary value problem for HIV evolution model. CEUR Workshop Proceedings, 2016; 1638: 508-514. DOI: 10.18287/1613-0073-2016-1638-508-514

\section{Introduction}

Singularly perturbed equations arise in the mathematical modeling of processes in chemical kinetics, biology, physiology and other areas of science. For problems of this type the methods, which give an asymptotic representation of solution, are successfully applied. The aforesaid is especially true for mathematical models of evolution biology, where an extremely slow biological evolution process proceeds against the background of significantly faster interactions of different nature. In this paper, the method of boundary functions is used for constructing asymptotic expansions of the solution to a singularly perturbed system of integro-differential equations with small parameter multiplying derivative.

\section{$2 \quad$ Model}

Let us consider the system of partial integro-differential equations

$u_{t}=b-u \int_{0}^{\infty} \beta(s) v(t, s) d s-q u$,

$v_{t}=\mu v_{s s}-m v+\beta u v$

with the initial and boundary conditions 
$u(0)=u^{0}, v(0, s)=v^{0}(s), v_{s}(t, 0)=0, v(t, \infty)=0$.

This system is a mathematical model of HIV evolution in a continuous phenotype space (see [1]). It is a result of the development of the in vivo dynamic model AIDS-1 proposed in [2]. The system (1) describes interactions between uninfected target cells of concentration $u(t), c e l l / \mathrm{mm}^{3}$, and infected cells with the density distribution

$v(t, s)$, cell $/ \mathrm{mm}^{3}$. Correspondingly, $V(t)=\int_{0}^{\infty} v(t, s) d s, \mathrm{cell} / \mathrm{mm}^{3}$, is the total concentration of infected cells. In the framework of this model a phenotype space is assumed to be continuous and one-dimensional ( $s \in[0,+\infty)$ - a dimensionless quantity). There is a continuous influx of the target cells (from the thymus, where they mature) at a rate $b, \mathrm{cell} /\left(\mathrm{mm}^{3} \cdot d a y\right)$. They die of natural reasons unrelated to the virus infection at a rate $q u$, where $q>0,1 /$ day. Parameter $\beta(s)=a s, \mathrm{~mm}^{3} /($ cell $\cdot$ day $)$, $a>0$, can be interpreted as the efficiency of a single virus particle in infecting a target cell. Infected cells die as a result of the infection or natural reasons at a rate $m v$, where $m=m(s)>0,1 /$ day. The average life spans of the uninfected and infected cells are $1 / q, 1 / m$ respectively. Random mutations are described by the dispersion $\mu v_{s s}, \mu>0,1 /$ day.

Without loss of generality and for simplicity it is assumed that only one of the parameters, $\beta(s)$, depends on $s$, and $m=m^{*}$ for all phenotypes. Besides although the model is formulated for $s \in[0,+\infty)$, usually $s$ is considered to be belong to a finite interval $[0, l], l=10$, for a given normalization and the condition $v(t, \infty)=0$ is replaced by $v_{s}(t, l)=0$.

Introducing dimensionless variables and parameters in the same way as in [3], the system (1) and conditions (2) can be written in the form

$$
\begin{aligned}
& \varepsilon u_{t}=b-u \int_{0}^{\ell} \beta v d s-u, \\
& v_{t}=v_{s s}-m v+p \beta u v, \\
& u(0)=u^{0}, v(0, s)=v^{0}(s), v_{s}(t, 0)=0, v_{s}(t, \ell)=0 .
\end{aligned}
$$

In (3) $\varepsilon=\sqrt{\mu m^{*}} / q \sim 10^{-3}$ for HIV, thereby this system is singularly perturbed system.

\section{Reduced system}

Setting $\varepsilon=0$ in (3), we obtain the degenerate (or reduced) system $\bar{v}_{t}=-m \bar{v}+p \beta \bar{u} \bar{v}+\bar{v}_{s s}$, $\bar{u}=1 /\left(1+\int_{0}^{\ell} \beta \bar{v} d s\right)$,

with initial and boundary conditions 
$\bar{v}(0, s)=v^{0}(s), \bar{v}_{s}(t, 0)=0, \bar{v}_{s}(t, \ell)=0$.

It should be noted that the solution $\bar{u}(t)$ of this system in general does not satisfy the initial condition in (4). But the solution of associated system (where $v$ is a parameter)

$\tilde{u}_{\tau}=-\left(1+\int_{0}^{l} \beta v d s\right) \tilde{u}+1$,

is $\tilde{u}(\tau)=\left(u^{0}-1 / f\right) \exp (-f \tau)+1 / f \rightarrow 1 / f$ as $\tau \rightarrow+\infty, f=1+\int_{0}^{l} \beta(s) v^{0}(s) d s$, that is the isolated root $u=1 /\left(1+\int_{0}^{l} \beta v d s\right)$ is an asymptotically stable rest point of system (7) and the initial value $u^{0}$ belongs to the domain of attraction of this root. In [4] it is shown that there is a passage to the limit

$$
\begin{aligned}
& \lim _{\varepsilon \rightarrow+0} u(t, \varepsilon)=\bar{u}(t), \quad 0<t \leq T, \\
& \lim _{\varepsilon \rightarrow+0} v(t, s, \varepsilon)=\bar{v}(t, s), \quad 0 \leq t \leq T, \quad 0 \leq s \leq l .
\end{aligned}
$$

Here $\bar{u}(t), \bar{v}(t, s)$ are the solutions of (5), (6) and $u(t, \varepsilon), v(t, s, \varepsilon)$ are the solutions of (3), (4).

Figure 1 shows the results of numerical simulations for the full and reduced systems (the thin line represents the plots corresponding to the full system (3), and the bold line represents the plots corresponding to the reduced system (5)). As we see, the solutions for the reduced and full systems agree fairly everywhere except a relatively short transition. For convenience of comparison results in this figure are shown for physical dimensional variables.
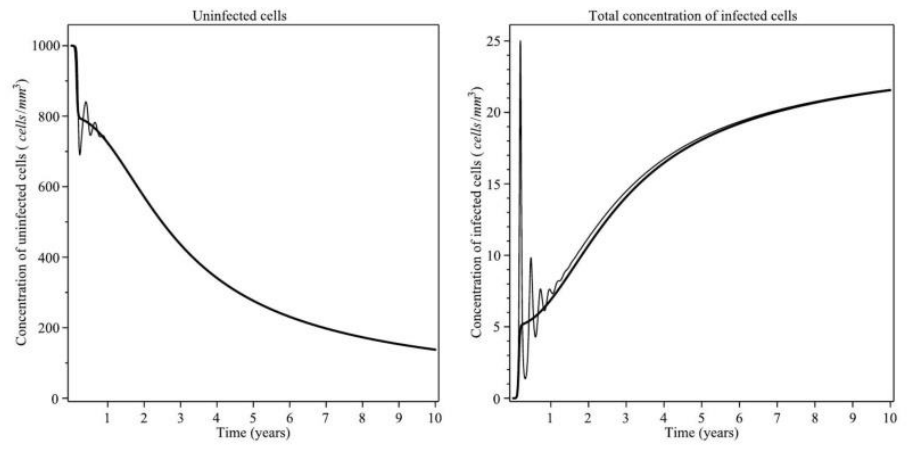

Fig. 1. Solutions of the full system (3) and reduced system (5)

If the initial condition is taken on or near the slow manifold, then the results obtained for both systems, full and reduced, coincide everywhere (see Fig. 2).

Using the approach developed in [5], we can prove the existence and uniqueness of degenerated problem (5), (6). 

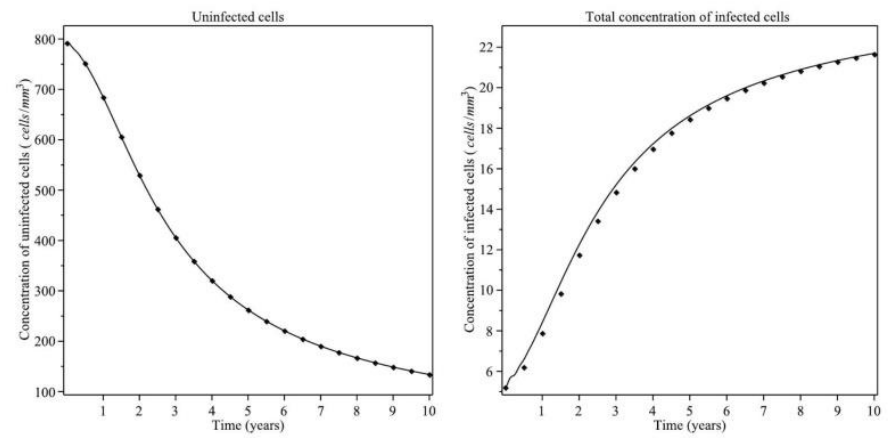

Fig. 2. Solutions of the full (thin line) and reduced (markers) systems, when initial point is taken on the slow manifold

\section{$4 \quad$ Asymptotic expansions}

Let us find the solution to problem (3), (4) in the form of asymptotic expansions in powers of small parameter $\varepsilon$. As we mentioned above, the solution of the degenerate system $\bar{u}(t)$ does not satisfy initial condition in (4) and, therefore, it may be assumed the existence of a boundary layer structure in the solution as $\varepsilon \rightarrow 0$. Following [6-9], in accordance with the method of boundary functions [10,11], such a solution can be found as a sum of a regular and boundary-layer series

$$
\begin{gathered}
u(t, \varepsilon)=\bar{u}(t, \varepsilon)+\Pi u(\tau, \varepsilon), \\
v(t, s, \varepsilon)=\bar{v}(t, s, \varepsilon)+\Pi v(\tau, s, \varepsilon),
\end{gathered}
$$

where $\bar{u}(t, \varepsilon)=\sum_{k=0}^{\infty} \varepsilon^{k} u_{k}(t), \bar{v}(t, s, \varepsilon)=\sum_{k=0}^{\infty} \varepsilon^{k} v_{k}(t, s)$ are the regular parts of the asymptotic expansions, $\Pi u(\tau, \varepsilon)=\sum_{k=0}^{\infty} \varepsilon^{k} \Pi_{k} u(\tau), \Pi v(\tau, s, \varepsilon)=\sum_{k=0}^{\infty} \varepsilon^{k} \Pi_{k} v(\tau, s)$ are the boundary-layer parts, and $\tau=t / \varepsilon$ is the boundary-layer variable. Formally substituting series (9) into equations (3) and the initial and boundary conditions (4) and equating the regular and boundary-layer parts (taking into account that $\varepsilon d / d t=d / d \tau)$, we obtain the equations

$$
\begin{aligned}
& \varepsilon u_{t}=1-\bar{u}-\bar{u} \int_{0}^{\ell} \beta \bar{v} d s, \\
& \Pi u_{\tau}=-\left(1+\int_{0}^{\ell} \beta(\bar{v}+\Pi v) d s\right) \Pi u-\bar{u} \int_{0}^{\ell} \beta \Pi v d s, \\
& \bar{v}_{t}=-m \bar{v}+p \beta \bar{u} \bar{v}+\bar{v}_{s \bar{s}}, \\
& \Pi v_{\tau}=\left[-m \Pi v+p \beta(\bar{u} \Pi v+\bar{v} \Pi u+\Pi u \Pi v)+\Pi v_{s s}\right] \varepsilon,
\end{aligned}
$$




$$
\begin{aligned}
& \bar{u}(0, \varepsilon)+\Pi u(0, \varepsilon)=u^{0}, \\
& \bar{v}(0, s, \varepsilon)+\Pi v(0, s, \varepsilon)=v^{0}(s), \\
& \bar{v}_{s}(t, 0, \varepsilon)=0, \quad \Pi v_{s}(\tau, 0, \varepsilon)=0, \\
& \bar{v}_{s}(t, \ell, \varepsilon)=0, \quad \Pi v_{s}(\tau, \ell, \varepsilon)=0 .
\end{aligned}
$$

It should be noted that regular terms in the right-hand sides of equations for boundary functions in (10) are calculated at $t=\varepsilon \tau$.

Regular terms $u_{0}(t), v_{0}(t, s)$ are the solutions of degenerate problem (5), (6). $\Pi_{0} u$ is the solution of initial value problem

$$
\begin{aligned}
& \Pi_{0} u_{\tau}=-\left(1+\int_{0}^{\ell} \beta v^{0} d s\right) \Pi_{0} u, \\
& \Pi_{0} u(0)=u^{0}-u_{0}(0),
\end{aligned}
$$

namely $\Pi_{0} u(\tau)=\left[u^{0}-u_{0}(0)\right] \exp \left[-\left(1+\int_{0}^{\ell} \beta v^{0} d s\right) \tau\right]$.

$\Pi_{0} v(\tau, s)=0$ can be found from the corresponding equation and the fact that this function is the boundary-layer function.

To construct more accurate approximations of the solution to the full system, it is necessary to use higher order asymptotic expansions. Expanding $\bar{u}(\tau \varepsilon, \varepsilon), \bar{v}(\tau \varepsilon, s, \varepsilon)$ in Taylor series around $\varepsilon=0$

$$
\begin{gathered}
\bar{u}(\tau \varepsilon, \varepsilon)=\sum_{k=0}^{\infty} \varepsilon^{k} A_{k}(\tau), \quad \bar{v}(\tau \varepsilon, s, \varepsilon)=\sum_{k=0}^{\infty} \varepsilon^{k} B_{k}(\tau, s), \\
A_{k}(\tau)=\sum_{r=0}^{k} \tau^{k-r} u_{r}^{(k-r)}(0), \quad B_{k}(\tau, s)=\sum_{r=0}^{k} \tau^{k-r} \frac{\partial^{k-r} v_{r}}{\partial t^{k-r}}(0, s)
\end{gathered}
$$

and substituting the resulting expansions into equations (3) and conditions (4), we equate the coefficients multiplying equal powers of $\varepsilon$ and find $k$-th, $(k \geq 1)$, terms of the asymptotic expansions according to the following scheme.

- Find $\Pi_{k} v(\tau, s)$ from the equation

$$
\begin{gathered}
\Pi_{k} v_{\tau}=\beta \sum_{l=0}^{k-1}\left(A_{l} \Pi_{k-l-1} v+B_{k-l-1} \Pi_{l} u+\Pi_{l} u \Pi_{k-l-1} v\right)- \\
-m \Pi_{k-1} v+\Pi_{k-1} v_{s s}
\end{gathered}
$$

and the condition $\Pi_{k} v(\infty, s)=0, \quad \forall s \in[0, \ell)$.

- $u_{k}(t), v_{k}(t, s)$ are the solution of the equations 


$$
\begin{gathered}
u_{k}=-\frac{u_{k-1 t}+\sum_{l=0}^{k-1} u_{l} \int_{0}^{\ell} \beta v_{k-l} d s}{1+\int_{0}^{\ell} \beta v_{0} d s}, \\
v_{k t}=-m v_{k}+p \beta u_{0} v_{k}+v_{k s s}+p \beta \sum_{l=0}^{k-1} u_{k-l} v_{l},
\end{gathered}
$$

satisfying the conditions $v_{k}(0, s)=-\Pi_{k} v(0, s), v_{k s}(t, 0)=0, v_{k S}(t, \ell)=0$.

- $\Pi_{k} u(\tau)$ is the solution of initial value problem

$$
\begin{aligned}
\Pi_{k} u_{\tau}=-(1 & \left.+\int_{0}^{\ell} \beta v^{0} d s\right) \Pi_{k} u-\sum_{l=0}^{k-1}\left(A_{l} \int_{0}^{\ell} \beta \Pi_{k-l} v d s+\right. \\
& \left.+\Pi_{l} u \int_{0}^{\ell} \beta\left(B_{k-l}+\Pi_{k-l} v\right) d s\right),
\end{aligned}
$$

The solution to this problem is always the boundary-layer function.

It should be noted that only zeroth regular terms of the asymptotic expansions are obtained from nonlinear equations. Terms of higher order approximations can be found from linear equations.

The asymptotic character of the expansions is justified as described [6-11].

\section{Conclusion}

Mathematical models in evolution biology should necessary combine the processes, specific time scales of which differ by several orders of magnitude. Accordingly such models are postulated in the form of the singularly perturbed systems of equations. The results obtained for relatively simple model can be naturally extended to much more complex models of evolution biology.

\section{Acknowledgements}

This work is supported by the Russian Foundation for Basic Research (grant № 1401-970-18-a) and the Ministry of education and science of the Russian Federation in the framework of the implementation of Samara University for 2013-2020 years and in the framework of the basic part of the state assignment (project № 214).

\section{References}

1. Korobeinikov A, Dempsey C. A continuous phenotype space model of RNA virus evolution within a host. Math. Biosci. Eng., 2014; 11(4): 919-927.

2. Nowak MA, May RM. Virus Dynamics: Mathematical Principles of Immunology and Virology. Oxford Univ. Press, 2000. 
3. Archibasov AA, Korobeinikov A, Sobolev VA. Asymptotic expansions of solutions in a singularly perturbed model of virus evolution. Comput. Math. Math. Phys., 2015; 55(2): 240-250.

4. Archibasov AA, Korobeinikov A, Sobolev VA. The passage to the limit in a singularly perturbed system of partial integro-differential equations. Diff. Eq. [in print]

5. Dan Henry. Geometric Theory of Semilinear Parabolic Equations. Berlin, Springer-Verlag, New York, Heidelberg, 1981.

6. Nefedov NN, Nikitin AG, Urazgil'dina TA. The Cauchy problem for a singularly perturbed Volterra integro-differential equation. Comput. Math. Math. Phys., 2006; 46(5): 768-775.

7. Nefedov NN, Nikitin AG. The Cauchy problem for a singularly perturbed integrodifferential Fredholm equation. Comput. Math. Math. Phys., 2007; 47(4): 629-637.

8. Nefedov NN, Nikitin AG. The initial boundary value problem for a nonlocal singularly perturbed reaction-diffusion equation. Comput. Math. Math. Phys., 2012; 52(6): 926-931.

9. Nefedov NN, Omel'chenko OE. Boundary-layer solutions to quasilinear integrodifferential equations of the second order. Comput. Math. Math. Phys., 2002; 42(4), 470482.

10. Vasilieva AB, Butuzov VF, Kalachev LV. The Boundary Function Method for Singular Perturbation Problems. Philadelphia, SIAM, 1995.

11. Vasil'eva AB, Butuzov VF. Asymptotic Expansions of Solutions to Singularly Perturbed Equations. Moscow: Nauka, 1973. [in Russian] 